\title{
REBUILDING WITH A HEART: ARCHITECTURE ROLES IN POST-DISASTER PSYCHOSOCIAL INTERVENTIONS
}

\author{
Gloryrose Alcoran DY ${ }^{1}$, Maria Lovella Parcon NACES ${ }^{2}$ \\ 1ICEA2014 Secretariat, Petra Christian University, Surabaya, Indonesia, 2 Switowins, \\ Inc. - Knowledge Management and Communication Department, 27c Galaxy St. GSIS heights, \\ Matina, Davao City, Davao City, 8000 Philippines \\ E-mails: ${ }^{1}$ gloryrosedy@gmail.com; ${ }^{2}$ mlpnaces@gmail.com
}

Received 26 May 2015; accepted 25 February 2016

\begin{abstract}
The presented paper focuses on the role of architecture in post-disaster humanitarian efforts particularly on the return to normalcy and sustainable recovery. Two major approaches were introduce, 'Architecture of empowerment' and 'Strengths-based approach' to analyze two cases of post disaster rebuilding projects employed in Batug, Leyte Philippines after super-typhoon Haiyan. The objective of the paper was to formulate a framework for post-disaster humanitarian efforts in order to achieve sustainable recovery. Another objective was to look at the interrelationship of the humanitarian architecture and psychosocial intervention or the journey to normalcy. Key Informant Interviews with locals, observers and participants of the shelter project, were used to gather information for both projects. Qualitative analysis and descriptive method were used for the interpretation the gathered data. In the end, a framework was formulated which points out that the integration with the roles of humanitarian architecture ( provider, supporter and catalyst) with strength-based approaches in post-disaster efforts factors of resources, competencies and sustainable recovery and the interplay of all these factors are the vital elements in order to have successful rebuilding projects after disasters.
\end{abstract}

Keywords: post-disaster humanitarian efforts, empowerment, rebuilding, architecture, humanitarian architecture, emphatic, psychosocial.

\section{Introduction}

Every now and then, man-made and natural disasters occur. The United Nations International Strategy for Disaster Reduction called for the different nations to unite in order to aid those who are affected by the disasters. Globally, professionals and experts alike have been gathered to provide short and long term help for disasters. This is because in the past decades, disasters worldwide have increased in frequency and intensity. In 2013 alone, the Philippines experienced major disasters, earthquake in Bohol and super typhoon in Leyte. Munich Re, National Hazards Center at Kansas State University has mapped out disasters and revealed that disasters worldwide have increased in frequency and intensity over the past decades (The World Bank 2010).

Lessons learned from all of these disasters encouraged professionals to collaborate with whole populations, public officials, business leaders, residents, professio- nals, etc. to not only rebuild but to also rehabilitate the disaster stricken society. Emiko Okoyama, Mayor of Sendai, Japan, stated that the intact collaboration of various professionals rather than separate approaches strengthened their swift resilient recovery after 2011 tsunami disaster. In October 2012, UNISDR recognized Okoyama's city for its role model strategy for rehabilitation and post-disaster recovery ( $\mathrm{PwC} 2013$ ).

While natural disasters are increasing and humanitarian agencies, professionals are increasingly being called upon to join forces to provide post-disaster responses, the involvement of the architectural profession is surprisingly low. In this collaboration of post disaster response, architects were often the last people needed in disaster reconstruction, says Architect David Sanderson, director of the Centre for Development and Emergency Practice in the department of architecture, Oxford Brookes University. The roles of architects, 
he said were at best, minimal in these circumstances because they were taught to focus more on the infrastructure rather than the people. This according to Sanderson is contrast to what is needed in disaster situation. The seemingly marginal contribution of the architectural sector stems from the common concept that what they do are mainly for the aesthetics purpose of infrastructures. Therefore they were not prioritized in terms of providing immediate assistance in post disaster situations. Sanderson added, for architects, ownership of the design rests with them and fellow professionals; architects for rebuilding with a heart, on the other hand, looks in to the design rests with them and the beneficiaries (Jopling 2009).

Instead of focusing on what skills the architect can contribute to the humanitarian sector, it is more important to consider what humanitarian sector needs from skills of the architectural industry. One of the key issues raised during the interview for post-disaster shelter and the role of the building professional, was that the beneficiaries who are important players in post disaster reconstruction, were overlooked (Cage et al. 2009).

In the aftermath of the disasters, professionals have gather and provide their own expertise in post disaster recoveries. Though they may be experts in their professions, their views might not fit well with the locals considering that these external experts, more often than not, are unfamiliar to the area. Engaging beneficiaries through sharing and cooperation is an essential part in long term response projects such as rebuilding of infrastructure program. Tovivich's Architecture of Empowerment, Research espoused theory and theory-in-use emphasized the need for collaboration. Humanitarian architecture takes a different design process compared to the traditional one in the sense that the design process of architects includes participation with the community it tries to help (Tovivich 2010). This is because rebuilding infrastructures and provision of shelter does not refer to provision of temporary tents or spaces with waterproof canvas as roof rather it is far more complex. The rebuilding of a disaster stricken community needs an emphatic approach, in which involvement of the local people is imperative. The views, the concepts and experience-based knowledge of their local environment combined with the knowledge and expertise of professionals, are necessary in order to rebuild infrastructures that will help people rehabilitate and hasten their return to normalcy.

\section{Understanding the architect's role in natural recovery and return to normalcy}

Humanitarian efforts in post disaster assistance, pose a challenge to conventional architecture as the discipline has long been associated with the upper class population and deemed as extravagant and unnecessary. But as the number of disasters increase by the decade, vital roles of architects in long-term post disaster recovery gradually emerged and emphasized in occurring studies. Alternative architecture practice through humanitarian architecture can help communities through the application of professional knowledge in terms of rebuilding the external surrounding and contribute to the psychosocial recovery of survivors.

In Figure 1, the natural recovery and return to normalcy of the survivors can be initiated through making most out of provided and available resources and the competencies. Resources are the staple needs in all possible activities initiated. In post disaster humanitarian efforts, resources are limited therefore, what little resource available must be maximized. In terms of competencies, the community participation, empowerment and engagement are imperatives in sustainable recovery. This is a strength based approach encouraging a more integrative community. With the theoretical understanding and smart recommendations provided by the professionals added to the grassroots knowledge of the local people about their environment and culture, resources can be efficiently mobilized and effectively used towards sustainable recovery (Mooney et al. 2011).

In this strength based approach, the architect can contribute to the post-disaster humanitarian effort through implementing their skills in the social system adjustments of the people, contributing significantly to the competencies present in the communities. In this humanitarian sense, architects can make recommendations through their expertise in creating valuable designs based on local structures and environment. Architect combines technical, practical and management skills working as a creative thinker that can provide a tailor fit design based on the environment and the community culture (Cage et al. 2009).

The design expands to understanding infrastructures, to landscapes, economics and the people involved. The humanitarian architect knows how to make designs of the infrastructure in harmony with the local culture. This can provide a guide on how to maximize resources and empower the community. These are the skills and knowledge that the post disaster sector needs.

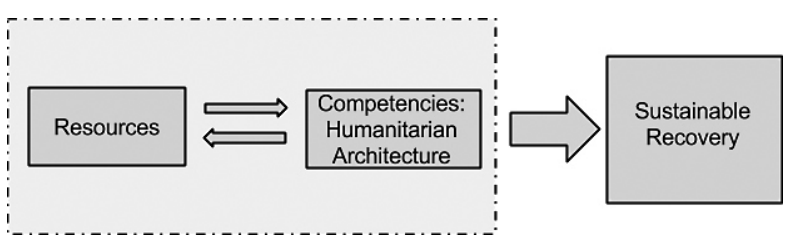

Fig. 1. Strength Based Approach (Mooney et al. 2011) 
The interchange of knowledge between the locals and experts enables all parties involved to operate an post disaster recovery activity that could be sustained longer.

\section{Three roles of architecture empowerment}

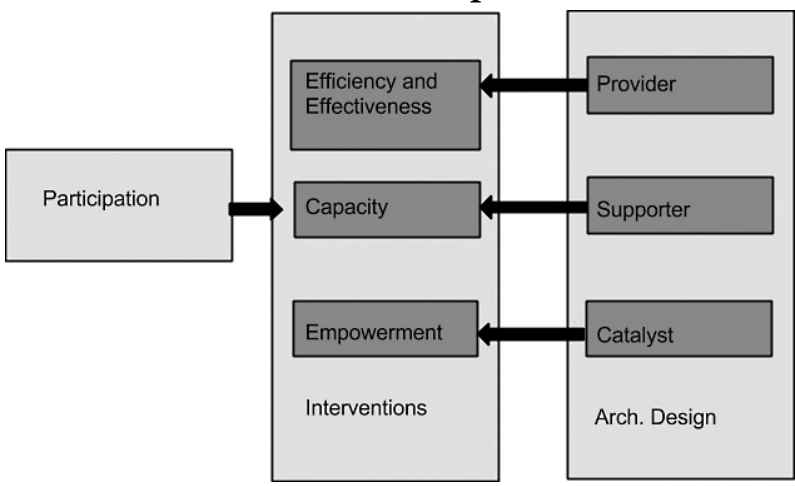

Fig. 2. Architecture of Empowerment ${ }^{1}$

Supitcha Tovivich's “Architecture of Empowerment" Research espoused theory and theory-in-use perspective explores this new architectural professionalism and explains the humanitarian architectural design process. In Tovovich's "Architecture of Empowerment", participation is the key element in the skills and knowledge sets of the new architectural practice in order to arrive with an efficient and effective design as well as empowered and capacitated stakeholders. (See Tovivich 2010).

The provider takes on the role for efficiency and effectiveness whereby it makes sure that the product is implemented in line with the purpose of what it is meant for. The supporter is in-charge of technology transfer in order to capacitate the local community while the catalyst empowers the local community to take control of their situation through the application of their skills sets.

While it is emphasized that the values of the new architectural professionalism strongly practices the role of a catalyst and supporter in the architectural design process, in order for efficiency and effectiveness to be achieved, all factors involving product-process, efficiency and effectiveness, local capacity building and empowerment should be involved. In other words, there must be a strong balance between participation and empowerment between organizations and stakeholders and that there is an interconnection between these roles (Tovivich 2010).

Based on this approach, in order for architects to be humanitarian, they must take on roles that are both design and non-design.

\footnotetext{
1 Figure 2 shows the divergent relationship between the architect in the paradigm of the provider, supporter or catalyst in espoused theory. (Tovivich 2010)
}

\section{Methodology}

This paper discussed two post disaster shelter projects employed in Batug, Leyte after super-typhoon Haiyan. Key Informant Interviews with locals, observers and participants of the shelter project, were used to gather information for both projects. Qualitative analysis and descriptive method were used for the interpretation the gathered data.

Being a study with two cases, this research does not have a strong content validity and cannot establish universal causal relation between factors and the success and failures of the projects. Through Tovivich's "Architecture of Empowerment" Research espoused theory and theory-in-use perspective, in understanding role of humanitarian architecture towards sustainable recovery, this weakness of data study was given theoretical spine and direction for the analysis.

The angle for analysis focused on the integration of beneficiaries and architects efforts in the process of rebuilding their lives back to normalcy. It looked into beneficiary participation and the roles of the architect as provider, supporter and catalyst and how it strengthens the sustainability of the intervention through efficiency and effectiveness, capacity and empowerment.

It is not the goal of this paper to arrive at conclusions with universal agreement. Rather it would look into deeper explanation and understanding of the dynamics of human relations in Architecture and humanitarian efforts in long term post disaster shelter projects.

\section{Dulag Leyte: Project 'A" Bunker and Project 'B' Cub house}

In November 7, 2013, the super-typhoon Haiyan locally known as Yolanda hit eastern Samar. It caused a storm surge which affected 46 provinces and 14 million people. Documents revealed that there were over 6,000 total fatalities and over 1,700 people were reported missing making this Category 5 super typhoon, the deadliest typhoon to hit the country. In Tacloban, Philippines, around half a million houses were severely damaged, five million people were displaced. Humanitarian efforts initially focused more on lifesaving emergency shelter assistance in their post disaster relief assistance. This eventually shifted towards a self-recovery support.

\section{Project 'A' Bunker}

Professionals for the shelter cluster post disaster relief assistance gathered to address this situation. In the Experts Forum on Rebuilding Communities and Ecosystems post-Yolanda, one of the key gaps for the number of damaged and destroyed lives and infras- 
tructures was due to the lack of typhoon barriers in the region. It is through this context that Project ' $A$ ' Bunker, and local community organizer organized a multifunctional building that is approximately 300 sqm using the key concepts of 'Beyond Green' architecture.

Project 'A' Bunker Build started a project to construct an infrastructure with typhoon resistant design. A non-Filipino design build firm was the main facilitator of Project 'A' Bunker Build had no close contact with the locals of the site prior to building the Project 'A' Bunker. They had, however, close contact with the locals during the ten-day construction wherein the local volunteers of fourteen participated in an on-site construction and technology transfer. From February 21 to March 4, 2014 the Project 'A' Bunker Build, together with one hundred (100) volunteers from all over the world constructed the first ever typhoon resistant building at Dulag, Leyte. The technology transfer was participated by locals as well as national volunteers. The latter translated the details of the construction to the former since the local volunteers could not understand the medium used for communication which was English. The Project 'A' Bunker Build did not do participatory design in the initial phase of the schematic development of the building because there was already a design made by the foreign architect in place prior to building and presenting to the locals.

However, when asked if they will stay and use the Project 'A' Bunker, one of the key informant interviewees, said that he will use the Project 'A' Bunker for evacuation and expressed his desire construct his house with the same design. The Project 'A' Bunker technology transfer was an example of an on-site participatory building. While the construction was going on, the local volunteers from community were given the task to re-echo their construction activity to their community.

On the last day of construction, on the tenth day, they were given a chance to present their new knowledge to the entire crew and to the community as well.

Since the community did not play a vital role in the creation of the conceptual and schematic design of the Project 'A' Bunker and the planning of the entire area, the Project 'A'Bunker did not use the participatory design approach of architecture. It did use community building and participatory construction of the structure's development. The presence of the technology transfer and the community engagement in the decision process of the development were done in an attempt to pave way for the rehabilitation of the community.

Five months after the ten-day construction activity, the Project 'A' Bunker building remains unfinished. According to a local resident, the building was still how it looked like in March 4, 2014 when the international volunteers were done with their 10 day construction project. After the activity, there were no major improvements in its construction. The building remains nonfunctional.

\section{Project 'B' Cub house}

Another innovation to help the survivors in the post disaster shelter cluster of the Haiyan survivors is the Project ' $B$ ' Cub house projects initiated by the same local management as Project 'A' Bunker. Locals were taught to build their own houses constructed from materials found in their vicinity. Project ' $B$ ' Cub house project is an eco-designed village project, an innovative means to construct houses using cheap materials such as mud which are costless. The locals built houses from mud and straws together with volunteers from earth village. The Project 'B' Cub house project used participation design in architecture.

In the construction of each house, this project used full participation of the beneficiaries. From the conceptualization of the design to the final construction of the house, the beneficiaries worked with the volunteers and local architects in order to build a house that will suit their needs. Initially there were fourteen houses built and as of this writing, the local stated that there are already 20 houses finished. Beneficiaries still continue to independently build and beautify their houses though all of the volunteers who assisted them in its initial construction have left the area.

\section{Analysis of two cases}

\section{Resources}

Both projects were initiated by foreign funded organizations and implemented through the local community organized management which is run by volunteers. Both projects maximized resources and materials locally found. Both constructions were operated at minimum costs, using recycled materials. Project ' $A$ ' Bunker and Project ' $B$ ' Cub house project both had an on-site collaboration effort, wherein the beneficiaries were part of the on-site construction. It is important to note that the effort in the on-site construction of the Project 'A' Bunker was divided between a hundred non-locals volunteers and fourteen locals while Project ' $B$ ' Cub house construction effort divided between all the household members and one or two non-resident volunteers.

In terms of resources for construction, both projects have monetary resources. However construction of the Project 'A' Bunker did not continue because the locals did not know how to proceed with the construction. 
The project in-charge and those who were largely involved left the community. The technology transfer was not able to equip the locals with knowledge on how to continue independently. In the Project ' $B$ ' Cub house project, the locals were still able to continue on their own because they were largely involved in the entire duration of the project. The beneficiaries knew how to gather needed material resources and continue with the construction. They were capacitated to find resources and continue with the activity.

\section{Competencies}

The Project 'A' Bunker Build and Project 'B' Cub house employed similar process in its construction wherein the locals helped in building. Both projects also engaged the presence of a technical professional in the design of the houses. Based on the espoused theory that states the architect as a provider, both projects had a technical provider to serve as the agent for the effectiveness and efficiency of project. Indeed, both projects were efficiently and effectively built according to plan.

However, though Project 'A' Bunker had on-site collaboration effort with fourteen of the local beneficiaries, the design were adapted from a foreign concept which was immediately applied to the local community. The beneficiaries neither contributed to the conceptualization or the designing of the Project ' $A$ ' Bunker Building.

In terms of the architect as a supporter, the Project 'A' Bunker build and the Project 'B' Cub house project were able to capacitate the local community in building a Beyond Green design and Project ' $B$ ' Cub house respectively. The Project 'A' Bunker Build conducted technology transfer in a form of focus group discussions and presentations during the last day of the 10 days Project 'A' Bunker Build. However, this technology transfer activity was not enough, to equip the locals with adequate knowledge to continue with the construction after the volunteers left the area. After the 10day construction period of the 100 international volunteers and 14 locals, the Project 'A' Bunker building was left as it was. Local residents stated that they were still waiting for foreign agencies to provide help with its construction.

In the Project ' $B$ ' $C u b$ house project, the locals were capacitated in such a way that they were able to replicate the construction of the Project ' $\mathrm{B}$ ' Cub house even after the volunteers left.

As a catalyst, the technical professionals of the Project 'A' Bunker build was not able to fully develop empowerment among the locals because the construction period only lasted for 10 days and $90 \%$ of those involved in the activity were non-locals. There was less engagement between the local community with the technical professional and vice versa. As a result, the local community was not able to take control of the technology and was not able to apply new skills sets after the construction days ended.

By contrast, the Project 'B' Cub house technical professional was able to fully take advantage of empowerment by being present during the periods of the mud house builds. Additionally, the local beneficiaries of the Project ' $B$ ' Cub house were also able to take part in the design of their bunkers. This factor of participation in the entirety of the design and construction phases proved important in terms of ownership and empowerment of the structures.

In other words, the Project 'B' Cub house project employed the participatory design approach. The locals collaborated with the architects and experts in the conceptualization, designing up to the construction and further beautification of the Project ' $B$ ' Cub house. There were no age limits; all members of the households were trained to build their houses on their own. The locals became independent in construction of their own houses that even when the experts, volunteers and professionals left, the beneficiaries still continued to finish their houses. The beneficiaries stated that they can continue in the construction and maintenance of the house because they already know how to use locally found materials for their houses.

\section{Participation}

Community involvement from the on-set of the project throughout its implementation is vital to the sustainability of long-term projects. In active community participation, there is this feeling of control through a sense of ownership of a project addressing their pressing needs. Lack of collaboration with the beneficiaries, can sometimes lead to misunderstanding, misinterpretations and resources being wasted. In the aftermath of Hurricane Katrina in New Orleans, California, USA, Brad Pitt's MakeItRight Foundation initiated a shelter project to provide long term help to the survivor of the hurricane. To accomplish the goals of this project, Pitt invited high profile architects to design shelter need responses. These architects were the only professionals who designed the houses. The survivors did not participate in the conceptualization and the design. The finished shelters were criticized and were called "Alien Architecture" (Cage et al. 2009). The lack of the participation of the beneficiaries resulted to the houses becoming non-fit to the local structures of the community. The community needs were not known to the architects thus were not intertwined with the designs they created. 
Participation of the beneficiaries, on the other hand, was considered as the strongest success factor in the Scandinavian Housing Project, a government housing project in Dumaguete. The then councilor who was head of the housing project, former Councilor Glory Alarcon-Dy recounted its success story. At the on-set of this project, they did not employ humanitarian architecture approach. Rather, similar to MakeItRight Foundation post disaster shelter help, they hired architects to design the houses, and all the beneficiaries had to do were to occupy the house. Contrary to their expectations, these houses were abandoned after a few weeks. As project head, according to her narration, she had to device another approach. This time, together with professionals, experts, they employed humanitarian architecture and involved the beneficiaries from the conceptualization and designing of the infrastructure, up to the construction of the houses. This approach proved to be exceptionally successful. The beneficiaries not only stayed, they also expanded their help and expanded to build their community through constructing their own basketball court, and they also paved the road. Dy, emphasized the sense of empowerment she observed among people, once their ideas merged with the professionals were built. The sense of ownership and identity were strong in the creation of their houses. As a result, this specific approach is being replicated and used as model for other government housing project (G. Dy, personal communication, May 17, 2013).

\section{Conclusions}

Architects have a role in Post Disaster Humanitarian Efforts. Based on the framework of Strengths-based approach adapted from Mooney and Tovovich's "Architecture and Empowerment", the technical professional should be involved in the interplay of resources and competencies in order to arrive at sustainable recovery.

Resources means providing the ample resources and using recycled materials as construction materials of the structures. Competencies mean intervening using the three interconnected roles of the architect and beneficiaries, provider, supporter and catalyst. Sustainable recovery is when all of these are in place. It is also important to note that these elements, resources in addition to the competencies are imperative in the guide towards sustainable recovery.

\section{Recommendation}

This framework can be used in the further research understanding of the interventions during natural disasters and why some community rebuilding projects recover sustainably and are swift to return to normalcy and why other community rebuilding projects fail.

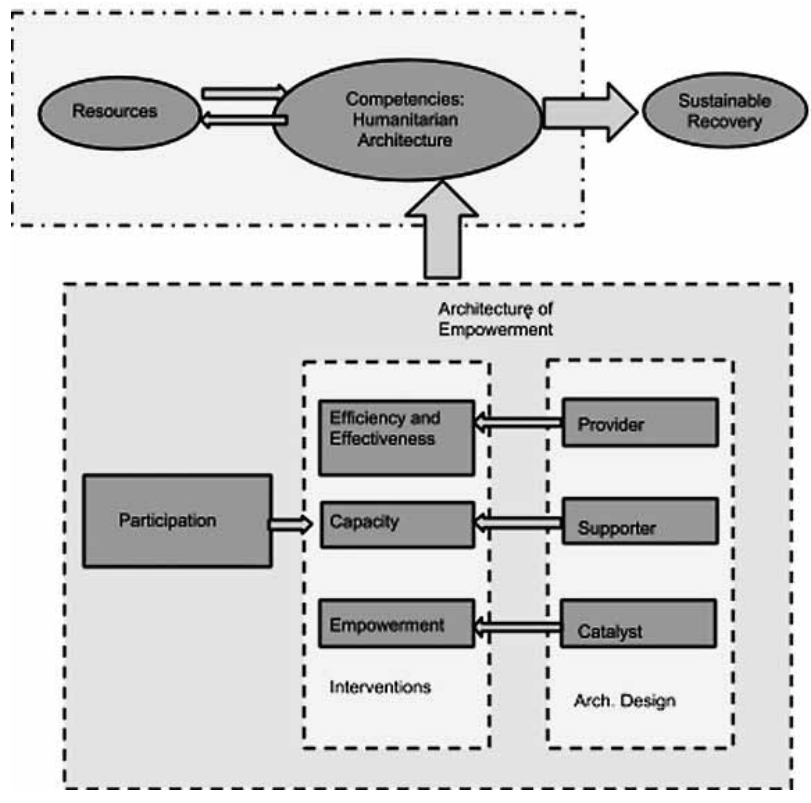

Fig. 3. Framework of architecture roles in post-disaster psychosocial interventions ${ }^{2}$

\section{References}

Cage, C.; Hingorani, D.; Jopling, S.; Parker, E. 2009. Building relevance: post-disaster shelter and the role of the building, inConference of the Centre for Development and Emergency Practice (CENDEP), 18 September 2009, Oxford, England. Oxford Brookes University and the International Federation of the Red Cross (IFRC).

Jopling, S. 2009. What does it take to operate as a development practitioner in post emergency shelter practice?: Master's thesis, Oxford Brookes University, Oxford, UK.

Mooney, M.; Paton, D.; De Terte, I.; Johal, Sarb.; Karanci, N.; Gardner, D.; Collins, S.; Glavovic, B.; Huggins, T.; Johnston, L.; Chambers, R.; Johnston D. 2011. Psychosocial recovery from disasters: a framework informed by evidence, New Zealand Journal of Psychology 40(4).

PwC. 2013. Rebuilding for resilience. Fortifying infrastructure to withstand disaster [online], [cited 19 December 2014]. PricewaterhouseCooperPwC. Available from Internet: http://www.pwc.com/en_GX/gx/psrc/publications/assets/ pwc-rebuilding-for-resilience-fortifying-infrastructure-towithstand-disaster.pdf

The World Bank. 2010. Damage, Loss and Needs Assessment [online], [cited 19 December 2014]. The International Bank for Reconstruction and Development/ The World Bank. Available from Internet: www.gfdrr.org/sites/gfdrr.org/files/ publication/Estimation\%20Volume3-WEB.pdf

Tovivich, S. 2010. Architecture for the urban poor, the 'new professionalism' of 'community architects' and the implications for architectural education: reflections on practice from Thailand: Doctoral Thesis. Development Planning Unit, University College London (DPU), London, UK.

\footnotetext{
2 Fig. 3 gives a possible conceptual framework of how architects may
} approach and operate a post -disaster psychological interventions 